\title{
LETTER
}

\section{Existing trial data do not support that hydroxyethyl starch is less harmful when given on more 'correct' indications}

\author{
Nicolai Haase, Rasmus G Müller and Anders Perner ${ }^{*}$ \\ Please see related research by Meybohm et al., http://ccforum.com/content/17/4/R166
}

Meybohm and colleagues hypothesise that so-called 'correct' administration of hydroxyethyl starch (HES) may be beneficial and may provide new guidelines for clinical use of HES, but this is not supported by trial data [1]. In contrast, subgrouping trials according to 'presumable correct indication' does not change the mortality estimate (see Figure 1). There is also very little statistical heterogeneity $\left(I^{2}\right)$ in the recent high-quality meta-analyses on HES indicating harmful effects independent of heterogeneity in trial design.

Several misinterpretations of the Scandinavian Starch for Severe Sepsis/Septic Shock trial publication [2] need correction. First, the exact dose of trial fluid was accounted for in 8,619 of 8,621 (99.98\%) trial patient-days. The lack of fluid data after day 3 is thus due to early death or discharge from the ICU.
Secondly, the amount of trial fluid did decrease over time because the percentages of patients who received trial fluid were $94 \%, 78 \%$ and $53 \%$ for days 1 to 3 , respectively. In fact, the majority of trial fluid was given within the first 38 hours because day 1 only lasted 14 hours (median). Finally, we cannot see the claimed 'conflicts between study protocol specifications and published baseline data, which also must be a misunderstanding.

We strongly discourage clinical algorithms for HES use, which are not supported by data from high-quality trials and which are against the recommendations by independent, scientific and regulatory organs [3-5]. Such algorithms for HES use must undergo testing in trials with low risk of bias before critically ill patients are exposed to potential harm.

\section{Authors' response}

Patrick Meybohm, Hugo Van Aken, Andrea De Gasperi, Stefan De Hert, Giorgio Della Rocca, Armand RJ Girbes, Hans Gombotz, Bertrand Guidet, Walter Hasibeder, Markus W Hollmann, Can Ince, Matthias Jacob, Peter Kranke, Sibylle Kozek-Langenecker, Stephan Alexander Loer, Claude Martin, Martin Siegemund, Christian Wunder and Kai Zacharowski

We are grateful to Haase and colleagues for their interest in our paper [1]. We appreciate their amendment to the original paper that 'lack of fluid data after day 3 is thus due to early death or discharge from the ICU' because this very important issue was not previously mentioned [2]. Further, we fully agree with the authors that 'such algorithms for HES use must undergo testing in trials with low risk of bias'.

\footnotetext{
* Correspondence: anders.perner@rh.regionh.dk

Department of Intensive Care, Copenhagen University Hospital - Rigshospitalet, DK-2100, Copenhagen, Denmark
}

Regarding the subgroup analysis, however, we disagree with the data analysis. Regarding 30-day mortality in the trial by Siegemund and colleagues, 36 out of 125 patients in the control group and 34 out of 119 patients in the HES group died (M Siegemund, personal communication), resulting in a risk ratio of 0.99 (0.67 to $1.4795 \%$ $\mathrm{CI})$. Taking these data into account would yield a combined risk ratio of 1.12 (0.88 to $1.4295 \% \mathrm{CI}$ ) in the subgroup of trials with 'high probability of correct fluid indication' (Figure 1). This combined point estimate for mortality does not indicate an increased risk of mortality in the subgroup of probably correct indication. The estimate rather suggests that, assuming the applied 


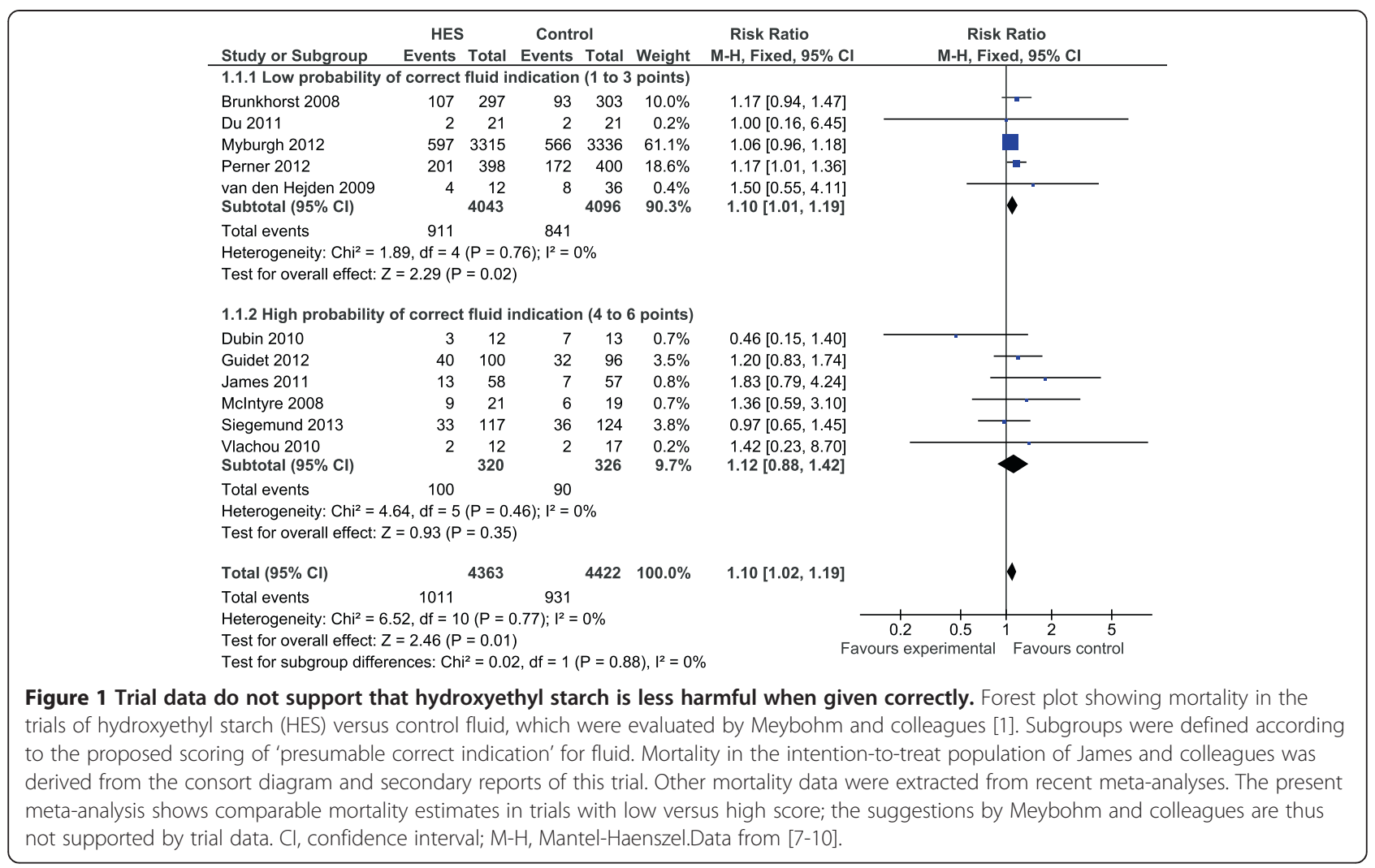

principles of fluid administration are adhered to, the tested interventions are associated with a similar risk for the outcome mortality.

Most importantly, rather than empirically splitting the trials into two groups according to a cutoff value of three points on the scale, we highly recommend performing meta-regression to investigate more appropriately how the different components of the scale did influence treatment effects [6].

In conclusion, algorithms for HES use must undergo testing in trials with low risk of bias, and identification of patients with correct indication is recommended.

\section{Abbreviations \\ HES: Hydroxyethyl starch.}

\section{Competing interests}

AP was the sponsor-investigator of the Scandinavian Starch for Severe Sepsis/Septic Shock (6S) trial, and NH was a member of the Steering Committee. The 6S trial was funded by the Danish Research Council, the Rigshospitalet Research Council, and the Scandinavian Society of Anaesthesiology and Intensive Care Medicine (the ACTA Foundation). B Braun Medical delivered trial fluid to all sites free of charge. Neither the funders nor B Braun Medical had an influence on the protocol, trial conduct, data analyses or reporting of the $6 \mathrm{~S}$ trial. AP is head of research in his department, which receives research funds from Fresenius Kabi, Germany, Cosmed, Italy and BioPorto Diagnostics, Denmark. B Braun Medical has covered his travel expenses for presenting $6 \mathrm{~S}$ trial data at the German Anaesthetic Congress 2012. RGM declares that he has no competing interests.
PM received lectures fees from Pulsion Medical Systems and independent research grants from B Braun Melsungen, Fresenius Kabi, Vifor Pharma, and CSL Behring. HVA received honoraria and travel expenses from Vifor Pharma, Abbott, and Fresenius Kabi. ADG received lecture/consultancy fees from Fresenius Kabi, CSL Behring and Grifols. HG held lectures for Fresenius Kabi, CSL Behring and Vifor Pharma. BG held lectures for Fresenius Kabi, received independent research grants from Fresenius Kabi, and is a member of the Grifols Albumin Advisory Board. MWH received lecture/consultancy fees and research grant support from B Braun Melsungen, Fresenius Kabi, and CSL Behring. Cl has received honoraria and independent research grants from Fresenius Kabi, Baxter Healthcare, and B Braun Melsungen. MJ has held lectures for Fresenius Kabi, Baxter, B Braun, Serumwerk Bernburg and CSL Behring, has received independent research grants from Serumwerk Bernburg, CSL Behring and Fresenius Kabi, and is member of the Grifols Albumin Advisory Board. PK received lecture fees from Fresenius Kabi and a research grant as the lead investigator (LKP) for the planning of a clinical trial investigating the initial haemodynamic stabilisation in severe sepsis. SK-L received honoraria for lectures, travel reimbursement and grants from $B$ Braun, Fresenius Kabi and CSL Behring. MS held lectures for Fresenius Kabi, B Braun and CSL Behring. CW received honoraria and independent research grants from Fresenius Kabi. KZ received consultancies, honoraria and grants from Fresenius Kabi, B Braun, and Vifor Pharma. The remaining authors declare that they have no competing interests.

Published: 15 Nov 2013

\section{References}

1. Meybohm P, Van Aken H, De Gasperi A, De Hert S, Della Rocca G, Girbes AR, Gombotz H, Guidet B, Hasibeder W, Hollmann MW, Ince C, Jacob M, Kranke P, Kozek-Langenecker S, Loer SA, Martin CD, Siegemund M, Wunder C, Zacharowski K: Re-evaluating currently available data and suggestions for planning randomised controlled studies regarding the use of hydroxyethyl-starch in critically ill patients - a multidisciplinary statement. Crit Care 2013, 17:R166. 
2. Perner A, Haase N, Guttormsen AB, Tenhunen J, Klemenzson G, Åneman A, Madsen KR, Møller MH, Elkjær JM, Poulsen LM, Bendtsen A, Winding R, Steensen M, Berezowicz P, Søe-Jensen P, Bestle M, Strand K, Wiis J, White JO, Thornberg KJ, Quist L, Nielsen J, Andersen LH, Holst LB, Thormar K, Kjældgaard A-L, Fabritius ML, Mondrup F, Pott FC, Møller TP, et al: Hydroxyethyl starch 130/0.42 versus Ringer's acetate in severe sepsis. N Engl J Med 2012, 367:124-134.

3. Dellinger RP, Levy MM, Rhodes A, Annane D, Gerlach H, Opal SM, Sevransky JE, Sprung CL, Douglas IS, Jaeschke R, Osborn TM, Nunnally ME, Townsend SR, Reinhart K, Kleinpell RM, Angus DC, Deutschman CS, Machado FR,

Rubenfeld GD, Webb S, Beale RJ, Vincent J-L, Moreno R: Surviving Sepsis Campaign: international guidelines for management of severe sepsis and septic shock, 2012. Intensive Care Med 2013, 39:165-228.

4. PRAC Recommends Suspending Marketing Authorisations for Infusion Solutions Containing Hydroxyethyl-starch. [http://www.ema.europa.eu/docs/en_GB/ document_library/Referrals_document/Solutions_for_infusion_containing_ hydroxyethyl_starch/Recommendation_provided_by_Pharmacovigilance Risk_Assessment_Committee/WC500144448.pdf].

5. FDA Safety Communication: Boxed Warning on Increased Mortality and Severe Renal Injury, and Additional Warning on Risk of Bleeding, for Use of Hydroxyethyl Starch Solutions in Some Settings. [http://www.fda.gov/ BiologicsBloodVaccines/SafetyAvailability/ucm358271.htm].

6. Jansen JP, Cope S: Meta-regression models to address heterogeneity and inconsistency in network meta-analysis of survival outcomes. BMC Med Res Methodol 2012, 12:152.

7. Zarychanski R, Abou-Setta AM, Turgeon AF, Houston BL, Mclntyre L, Marshal $J C$, Fergusson DA: Association of hydroxyethyl starch administration with mortality and acute kidney injury in critically ill patients requiring volume resuscitation: a systematic review and meta-analysis. JAMA 2013, 309:678-688.

8. Haase N, Perner A, Hennings LI, Siegemund M, Lauridsen B, Wetterslev M, Wetterslev J: Hydroxyethyl starch 130/0.38-0.45 versus crystalloid or albumin in patients with sepsis: systematic review with meta-analysis and trial sequential analysis. BMJ 2013, 346:f839.

9. James MF, Mitchell WL, Joubert IA, Nicol AJ, Navsaria PH, Gillespie RS: Resuscitation with hydroxyethyl starch improves renal function and lactate clearance in penetrating trauma in a randomized controlled study: the FIRST trial (Fluids in Resuscitation of Severe Trauma). $\mathrm{Br} J$ Anaesth 2011, 107:693-702.

10. James MFM, Michell WL, Joubert IA, Nicol AJ, Navsaria PH, Gillespie RS: Hydroxyethyl starch in patients with trauma. Br J Anaesth 2012, 108:160-161.

$10.1186 / \mathrm{cc} 13111$

Cite this article as: Haase et al:: Existing trial data do not support that hydroxyethyl starch is less harmful when given on more 'correct' indications. Critical Care 2013, 17:461 